\title{
Knowing when to stop
}

Axon guidance is not just a matter of sending axons in the right direction they must also terminate in the right place. The vertebrate retinotectal system is an excellent model for studying this mechanism, and John Flanagan and colleagues have developed an ingenious new in vitro assay to investigate how ephrin signals regulate the termination of retinal ganglion cell (RGC) axon growth in the tectum.

Axons from RGCs in the temporal hemisphere of the retina terminate in the anterior tectum, whereas axons from the nasal region terminate in the posterior tectum. Ephrin-A2 and -A5 are expressed in a low-anterior to high-posterior gradient across the tectum, and their receptors, the EphAs, are expressed in a hightemporal to low-nasal gradient in RGCs. Previous in vitro studies focused predominantly on how RGC axons make binary decisions, such as whether or not to grow on a stripe of ephrin-expressing tissue. However, Hansen et al. introduced a quantitative dimension by exposing RGCs from different nasal-temporal levels to varying ephrin concentrations.

The authors cultured explants of mouse retina (numbered $1-8$ to denote their position along the nasal-temporal axis) on membrane 'carpets' from various regions of the chick tectum. They found that both nasal and temporal RGC axons grew better on anterior than posterior tectal tissue. As might be expected, nasal axons grew better on posterior tectal tissue than did the temporal axons.

Next, the authors cultured retinal explants on carpets of membrane vesicles from cell lines that had been transiently transfected with an ephrin-A2 or ephrin-A5-expressing construct. They observed a smoothly graded response to ephrins across the retina. On ephrin-A5 carpets, explants from the nasal region (position 1) showed the most prolific axon outgrowth, whereas on ephrin-A2 carpets, outgrowth peaked at position 3 . Interestingly, this pattern mirrors the expression gradients of these molecules across the mouse superior colliculus (the mammalian equivalent of the tectum) - ephrin-A5 expression peaks at the posterior end, whereas ephrin-A2 is expressed in a biphasic gradient, peaking around the middle of the posterior half.

To quantify the response to ephrin-A2 further, the authors prepared vesicle carpets consisting of varying proportions of membranes from ephrin-A2-transfected cells and non-transfected anterior tectal cells. At the highest ephrin concentration (100\% ephrin-A2 membranes), there was no axon outgrowth from any of the retinal explants, whereas with 50\% ephrin-A2 membranes there was an abrupt switch from limited outgrowth to no outgrowth between the nasal and temporal halves. With 15\%
ephrin-A2 membranes there was a graded biphasic response, with maximal outgrowth occurring at retinal position 3 .

Intriguingly, there was little or no axon outgrowth from any of the explants with $0 \%$ ephrin-A2 membranes, indicating that low levels of ephrin-A2 have a growth-promoting effect on RGC axons. The positioning of RGC axons in the tectum is thought to require a counterbalance between attraction and repulsion, with the growth cones stopping where the two forces balance out. Hansen and colleagues' findings indicate that ephrins could provide both of these forces, and the data point to a model to explain how attraction turns to repulsion. The repellent actions of ephrins seem to depend on intracellular signalling, whereas ephrin-mediated attraction seems to rely on adhesion. Therefore, they propose that adhesion predominates at low ephrin concentrations, but as the levels increase, intracellular signalling pathways are activated, thereby generating a repulsive force that overrides the attraction.

Heather Wood

\section{(0) References and links} ORIGINAL RESEARCH PAPER Hansen, M. J. et al. Retinal axon response to ephrin-As shows a graded, concentration-dependent transition from growth promotion to inhibition. Neuron $\mathbf{4 2}$ 717-730 (2004)

FURTHER READING Wilkinson, D. G. Multiple roles of Eph receptors and ephrins in neural development. Nature Rev. Neurosci. 2, 155-164 (2001)

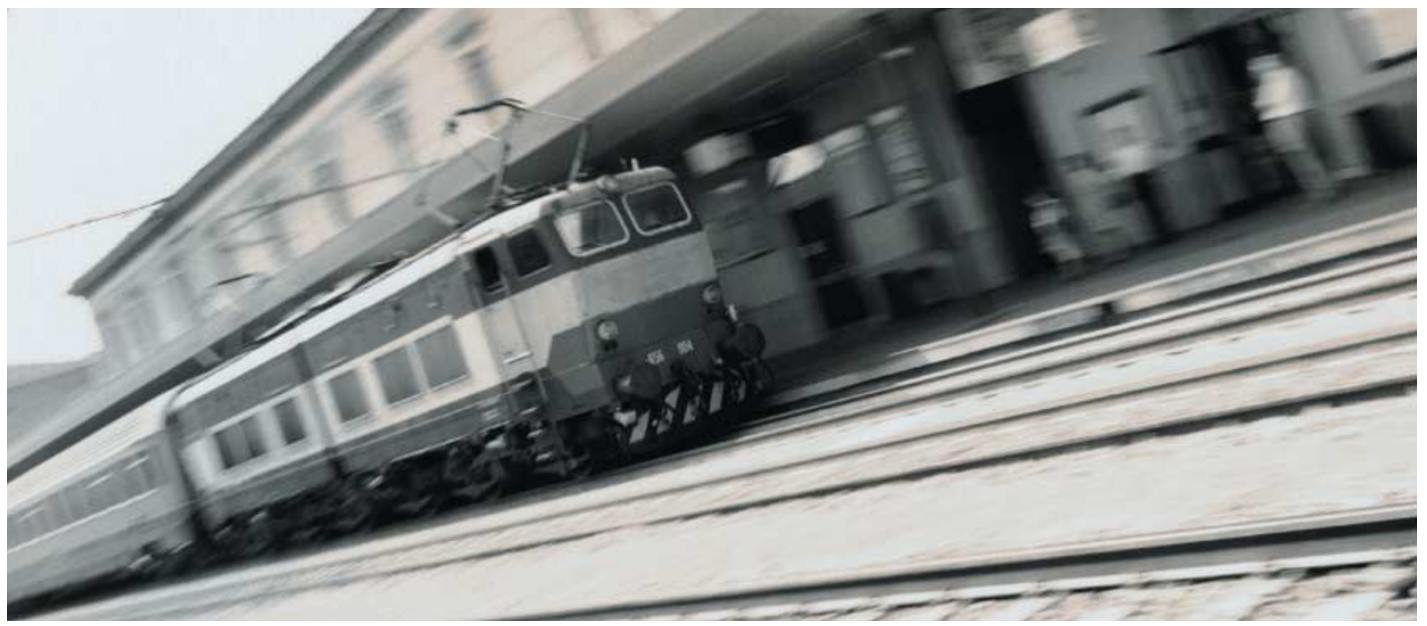

\section{IN THE NEWS}

Rico the wonder dog

Move over, Lassie - there's a new canine superhero in town. Rico the border collie might not have rescued his owner from a well, but as Julia Fischer's team at the Max-Planck Institute for Evolutionary Anthropology in Leipzig report in Science (11 June 2004), he has a remarkable way with words.

Not only can nine-year-old

Rico identify more than 200 objects by name, he is also capable of a form of reasoning called fastmapping, which was thought to be exclusive to humans.

This skill enables him to infer that an unfamiliar word corresponds to a new object, and he can still remember the object after being denied access to it for a month. According to The Telegraph (News online 11 June), "[Fischer and colleagues] believe they have shown something more than the intelligence of the breed, saying that seemingly complex language skills only seen before in children appear to be found in other species." However, some experts warn against crediting Rico with 'language skills'. Talking to $B B C$ News (11 June), behavioural ecologist Alex Kacelnik said, "We don't know that Rico has a particular ability to link sounds to objects, rather than link any arbitrary stimulus to objects. Rico probably has the general ability to connect things not a language ability."

Also, some dog owners remain unconvinced that obedience equals intelligence. Science writer James Gorman cites the example of a former family pet, a Pomeranian who "Would do anything, as long as there was food involved. When I thought I was teaching him to come when I called, by giving him a treat, what he learned was that if he ran away from me as often as possible, I would have to call him more often. That would mean more treats" (New York Times, 21 June).

Heather Wood 